\title{
Utilization of Del Nido Cardioplegia in Adult Coronary Artery Bypass Grafting
}

- A Retrospective Analysis -

\author{
Christian O’Donnell, BSc; Hanjay Wang, MD; Phat Tran, PhD; \\ Shari Miller, BSc; Paul Shuttleworth, BSc; Jack H. Boyd, MD
}

Background: Studies assessing the safety and effectiveness of Del Nido cardioplegia for adult cardiac surgery remain limited. We investigated early outcomes after coronary artery bypass grafting (CABG) using single-dose Del Nido cardioplegia vs. conventional multi-dose blood cardioplegia.

Methods and Results: The 81 consecutive patients underwent isolated CABG performed by a single surgeon. The initial 27 patients received anterograde blood cardioplegia, while the subsequent 54 patients received anterograde Del Nido cardioplegia. There were no differences in the baseline characteristics of each group nor any differences in the 30-day incidences of myocardial infarction, all-cause death, and readmission following surgery. The use of Del Nido cardioplegia was associated with shorter cardiopulmonary bypass time (98 vs. $115 \mathrm{~min}, \mathrm{P}=0.011$ ), shorter cross-clamp time ( $74 \mathrm{vs.} 87 \mathrm{~min}, \mathrm{P}=0.006$ ), and decreased need for intraoperative defibrillation ( $13.0 \%$ vs. $33.3 \%, P=0.030)$ compared with blood cardioplegia. To control for the difference in cross-clamp time, we performed propensity score matching with a logistical treatment model and confirmed that Del Nido cardioplegia provided similar outcomes as blood cardioplegia and also reduced the need for defibrillation independent of cross-clamp time.

Conclusions: Compared with conventional blood cardioplegia, Del Nido cardioplegia provided excellent myocardial protection with reduced need for intraoperative defibrillation, shorter bypass and cross-clamp times, and comparable early clinical outcomes for adult patients undergoing CABG.

Key Words: Cardioplegia; Cardiopulmonary bypass; Coronary artery bypass grafting; Defibrillation

D el Nido cardioplegia (DN) was originally developed in the early 1990 s at the University of Pittsburgh to optimize myocardial protection during congenital cardiac surgery, because immature pediatric hearts may be highly susceptible to ischemia-reperfusion injury and the damage caused by accumulation of intracellular calcium. ${ }^{1}$ Over the past decade, the use of DN has gradually expanded to the adult cardiac surgery arena because of its convenient ability to achieve up to $90 \mathrm{~min}$ of cardioplegic arrest with only a single dose, as compared with conventional multi-dose blood cardioplegia (BC), which must be given every $15-20 \mathrm{~min}$. Studies validating the safety and effectiveness of DN in adults, however, remain limited in number. ${ }^{2}$ Therefore, we examined the use of DN compared with BC for isolated coronary artery bypass grafting (CABG) operations performed by a single surgeon at our institution. We hypothesized that single-dose anterograde DN would provide myocardial protection and early clinical outcomes comparable to that of conventional multi-dose $\mathrm{BC}$ in adult CABG surgery.

\begin{abstract}
Methods
The protocols and policies followed in this study were approved by the Stanford University Institutional Review Board (IRB 38337). Because of the retrospective nature and minimal risk of our study, individual participant consent was waived. Our study used a validated 'before and after' group level study design to examine the effect of introducing Del Nido cardioplegia as the only variable that was changed in our CABG protocol. All patients who underwent isolated CABG performed by an experienced specialist in coronary revascularization surgery at our institution during a 2-year period from October 2014 to October 2016 were assessed for inclusion. In July 2015, our institution's perfusion protocol for CABG surgery was changed from using BC to solely using DN. Overall, 27 consecutive BC patients (October 2014 to July 2015) and 54 consecutive DN patients (August 2015 to August 2016) were identified. Re-operative procedures and minimally invasive direct coronary artery bypass surgeries, which
\end{abstract}

Received July 10, 2018; revised manuscript received October 18, 2018; accepted October 31, 2018; J-STAGE Advance Publication released online December 7, 2018 Time for primary review: 35 days

Department of Cardiothoracic Surgery, Stanford University School of Medicine, Stanford, CA, USA

Mailing address: Jack H. Boyd, MD, Clinical Assistant Professor, Department of Cardiothoracic Surgery, Stanford University School of Medicine, 300 Pasteur Drive, Stanford, CA 94305, USA. E-mail: jackboyd@stanford.edu

ISSN-1346-9843 All rights are reserved to the Japanese Circulation Society. For permissions, please e-mail: cj@j-circ.or.jp 
represent a significant proportion of the surgeon's practice, were excluded.

All patients received general anesthesia and underwent a median sternotomy. Cardioplegia was delivered anterograde at $4^{\circ} \mathrm{C}$ in all cases without any retrograde dosing. Patients in the $\mathrm{BC}$ group received an initial $1,000 \mathrm{~mL}$ bolus dose, followed by additional $200 \mathrm{~mL}$ doses of a $4: 1$ blood to crystalloid cardioplegia solution every $15-20 \mathrm{~min}$ as needed; patients in the DN group received a single $1,200 \mathrm{~mL}$ bolus of 1:4 blood to DN cardioplegia solution. Of note, an additional dose of DN $(1-10 \mathrm{~mL} / \mathrm{kg})$ was given at the surgeon's discretion to $6 \mathrm{DN}$ patients who required prolonged arrest for more than $90 \mathrm{~min}$. A component comparison of the DN and BC solutions used in our perfusion protocols is detailed in Table $\mathbf{1 .}$

The primary outcomes of this study were the 30-day incidences of all-cause death, stroke, and myocardial infarction (MI) after surgery. Secondary outcomes included cardiopulmonary bypass (CPB) time, cross-clamp time, need for intraoperative defibrillation, immediate post-CPB left ventricular ejection fraction (LVEF) and right ventricular (RV) function measured by transesophageal echocardiography (TEE), incidence of postoperative atrial fibrillation, length of hospital and intensive care unit (ICU) stay, and readmission rate.

\section{Statistical Analysis}

We used Stata version 14.2 (StataCorp, College Station, TX, USA). Continuous variables are expressed as mean \pm standard deviation, and comparisons were performed using two-sample t-test with unequal variance or a two-sample Wilcoxon rank-sum test. Categorical data are expressed as counts with percentages and analyzed by Pearson Chi-square or Fisher's exact test. Propensity score matching (PSM), based on the standard logistical treatment model (logit) with bias correction, was performed using the following variables: age, body mass index (BMI), sex, preoperative creatinine, diabetes, Society of Thoracic Surgeons (STS) 30-day risk score, pre-CPB LVEF measured by TEE, and cross-clamp time. The purpose of PSM was to control for preoperative characteristics and variables

\begin{tabular}{|lcc|}
\hline $\begin{array}{l}\text { Table 1. Comparison of DN and BC Composition per } 1 \mathrm{~L} \text { of } \\
\text { Base Solution (Plasma-Lyte A for DN and Plegisol } \\
\text { for BC) }\end{array}$ \\
Sodium & DN (1:4) & BC (4:1) \\
Potassium & $153 \mathrm{mEq}$ & $129.6 \mathrm{mEq}$ \\
Bicarbonate & $31 \mathrm{mEq}$ & $100 \mathrm{mEq}$ \\
Magnesium & $13 \mathrm{mEq}$ & $20 \mathrm{mEq}$ \\
Chloride & $19.24 \mathrm{mEq}$ & $32 \mathrm{mEq}$ \\
Calcium & $98 \mathrm{mEq}$ & $160 \mathrm{mEq}$ \\
Osmolality & 'Trace'a & $2.4 \mathrm{mEq}$ \\
Acetate & $294 \mathrm{mOsm}$ & $304 \mathrm{mOsm}$ \\
Gluconate & $27 \mathrm{mEq}$ & - \\
Mannitol 20\% & $32 \mathrm{mEq}$ & - \\
Lidocaine 1\% & $2 \mathrm{~g}$ & - \\
\hline
\end{tabular}

BC, blood cardioplegia; DN, Del Nido cardioplegia. a'Trace' calcium in Del Nido refers to the calcium content contained in the $20 \%$ of the patients' blood delivered with the solution.

such as cross-clamp time that may affect outcomes. The caliper width for propensity matching was restricted to 0.2 of the standard deviation of the logit to ensure optimal pair matching. The standard mean difference (SMD, d) was calculated for continuous variables as the difference in the mean of the treatment group and control group divided by the standard deviation of the samples. For binary variables, the logit ratio was used.

In total, $2 \mathrm{DN}$ and $2 \mathrm{BC}$ patients were excluded from PSM analysis because of lack of pre-CPB LVEF data. In addition, $1 \mathrm{DN}$ and $7 \mathrm{BC}$ patients were excluded from the PSM analysis because of the strict caliper size and number of preoperative characteristics. These included (1) a 76-year-old male DN patient with BMI 22.5, preoperative creatinine 1.4 , and pre-CPB LVEF 40\%; (2) a 67-year-old non-diabetic male BC patient with STS risk score $1.9 \%$ and pre-CPB LVEF 15\%; (3) a 63-year-old non-diabetic male BC patient with STS risk score $0.84 \%$ and pre-CPB LVEF $60 \%$; (4) a 61-year-old non-diabetic male BC patient with

\begin{tabular}{lccc|}
\hline \multirow{2}{*}{ Table 2. Study Patients' Demographics, Characteristics, and Comorbidities } & \\
\cline { 2 - 4 } \multicolumn{1}{c}{ Preoperative characteristics } & Unmatched & P value \\
Male & $20(74.1 \%)$ & DN $(\mathbf{n}=54)$ & 0.324 \\
Age (years) & $61.3 \pm 10.9$ & $45(83.3 \%)$ & 0.501 \\
BMI (kg/m $\left.{ }^{2}\right)$ & $27.0 \pm 4.5$ & $63.0 \pm 10.6$ & 0.405 \\
Diabetic & $13(48.2 \%)$ & $28.0 \pm 5.5$ & 0.529 \\
STS score (\%) & $1.3 \pm 1.4$ & $30(55.6 \%)$ & 0.721 \\
NYHA & & $1.2 \pm 1.1$ & \\
I & $11(40.7 \%)$ & & 0.751 \\
II & $8(29.6 \%)$ & $24(44.4 \%)$ & 0.736 \\
III & $4(14.8 \%)$ & $18(33.3 \%)$ & 1.00 \\
IV & $4(14.8 \%)$ & $9(16.7 \%)$ & 0.214 \\
Creatinine (mg/dL) & $1.1 \pm 0.3$ & $3(5.6 \%)$ & 0.733 \\
Pre-CPB LVEF (\%) & $1.1 \pm 0.9$ & 0.676 \\
IABP & $50.2 \pm 14.2$ & $51.6 \pm 12.1$ & 0.681 \\
\hline
\end{tabular}

BMI, body mass index; CPB, cardiopulmonary bypass; IABP, intra-aortic balloon pump; LVEF, left ventricular ejection fraction; NYHA, New York Heart Association; STS, Society of Thoracic Surgeons. Other abbreviations as in Table 1.

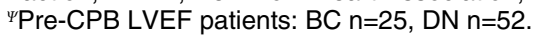




\begin{tabular}{|lccc|}
\hline \multicolumn{2}{l}{ Table 3. Early Outcomes (Unmatched Cohorts) } & Unmatched \\
\cline { 2 - 4 } Cardiac function & $\mathbf{B C}(\mathbf{n = 2 5})$ & $\mathbf{D N}(\mathbf{n}=\mathbf{5 2})$ & P value \\
Post-CPB LVEF (\%) & $53.0 \pm 12.5$ & $55.5 \pm 9.6$ & 0.435 \\
RV dysfunction & $1(4.0 \%)$ & $0(0 \%)$ & 0.325 \\
Medical outcomes & $\mathbf{B C}(\mathbf{n = 2 7})$ & $\mathbf{D N}(\mathbf{n = 5 4})$ & P value \\
Hospital stay (days) & $6.7 \pm 3.2$ & $6.8 \pm 4.6$ & 0.782 \\
ICU stay (days) & $3.0 \pm 2.3$ & $2.9 \pm 4.5$ & 0.251 \\
Creatinine (mg/dL) & $1.1 \pm 0.4$ & $1.2 \pm 0.8$ & 0.837 \\
Atrial fibrillation & $4(14.8 \%)$ & $12(22.2 \%)$ & 0.559 \\
Readmission & $4(14.8 \%)$ & $6(11.1 \%)$ & 0.724 \\
All-cause death & $0(0 \%)$ & $0(0 \%)$ & - \\
MI & $0(0 \%)$ & $0(0 \%)$ & - \\
Stroke & $1(3.7 \%)$ & $2(3.7 \%)$ & 1.000 \\
\hline
\end{tabular}

ICU, intensive care unit; MI, myocardial infarction. Other abbreviations as in Table 1.

\begin{tabular}{|lccl|}
\hline \multicolumn{2}{|c|}{ Table 4. Surgical Data (Unmatched Cohorts) } & \multicolumn{3}{l|}{ Unmatched } \\
\cline { 2 - 4 } \multicolumn{1}{c}{ Surgical factors } & BC $(\mathbf{n}=\mathbf{2 7})$ & DN $(\mathbf{n}=\mathbf{5 4})$ & P value \\
No. of bypasses & $3.0 \pm 0.7$ & $3.1 \pm 0.8$ & 0.746 \\
Cross-clamp time (min) & $87.4 \pm 20.0$ & $74.4 \pm 16.2$ & $0.006^{\star *}$ \\
CPB time (min) & $114.5 \pm 27.2$ & $98.3 \pm 22.6$ & $0.011^{*}$ \\
Need for defibrillation & $9(33.3 \%)$ & $7(13.0 \%)$ & $0.030^{*}$ \\
\hline
\end{tabular}

Abbreviations as in Tables 1,2.

STS risk score $0.56 \%$ and pre-CPB LVEF $68 \%$; (5) a 62 -year-old non-diabetic male BC patient with creatinine 1.2 and STS risk score $0.61 \%$; (6) a 58 -year-old male with BMI 24.7, preoperative creatinine 1.0, and STS risk score $0.38 \%$; (7) a 72-year-old non-diabetic female BC patient with BMI $21.6 \%$, STS risk score $1.8 \%$, and pre-CPB LVEF $81 \%$; and (8) a 41 -year-old male with creatinine 0.7 , STS risk score $0.26 \%$, and pre-CPB LVEF $37.5 \%$.

\section{Results}

\section{Unmatched Analysis}

The unmatched $\mathrm{DN}(\mathrm{n}=54)$ and $\mathrm{BC}(\mathrm{n}=27)$ groups were similar in all baseline characteristics, with comparable mean age (63.0 vs. 61.3 years, respectively), preoperative STS risk score (1.2\% vs. $1.3 \%$, respectively), preoperative creatinine (1.1 vs. $1.1 \mathrm{mg} / \mathrm{dL}$, respectively), pre-CPB LVEF (51.6\% vs. $50.2 \%$, respectively), and NYHA functional class (Table 2). As presented in Table 3, there were no significant differences in any of the primary outcomes of all-cause death $(0 \%$ in both groups), stroke (3.7\% in both groups), or postoperative MI ( $0 \%$ in both groups) at 30 days after surgery. Moreover, there were no significant differences between groups in post-CPB LVEF (DN 55.5\% vs. BC $53.0 \%$ ) or incidence of post-CPB RV dysfunction (DN $0 \%$ vs. BC 4.0\%). The incidence of postoperative atrial fibrillation, length of hospital and ICU stay, and readmission rate were also similar between the two groups.

The use of DN was associated with a significantly shorter CPB time (98.3 vs. $114.5 \mathrm{~min}, \mathrm{P}=0.011)$ and cross-clamp time (74.4 vs. $87.4 \mathrm{~min}, \mathrm{P}=0.006$ ) compared with using $\mathrm{BC}$ (Table 4). The need for defibrillation was also reduced significantly in the DN group compared with the $\mathrm{BC}$ group
( $13.0 \%$ vs. $33.3 \%, P=0.030)$. The Cohen's $\mathrm{d}$ effect size calculated the predicted practical significance of these reductions to have a medium to large effect (CPB time, $\mathrm{d}=0.67$; cross-clamp time, $\mathrm{d}=0.74)$.

\section{PSM Analysis}

$\mathrm{DN}$ cardioplegia was associated with a significant reduction in $\mathrm{CPB}$ and cross-clamp times compared with $\mathrm{BC}$ in the unmatched analysis. Therefore, we decided to use PSM to control for cross-clamp time, in addition to other standard preoperative baseline characteristics such as age, sex, BMI, diabetic status, preoperative creatinine, STS risk score, and LVEF. PSM was successfully performed with $51 \mathrm{DN}$ patients and $18 \mathrm{BC}$ patients. As before, there were no deaths or postoperative $\mathrm{MI}$ in either group, and the 30-day incidence of stroke was 3.9\% in the DN group vs. $5.6 \%$ in the $\mathrm{BC}$ group $(\mathrm{P}=0.764$, Table 5). The length of hospital and ICU stay, and rate of admission were also all similar between the matched groups.

After controlling for cross-clamp time (DN 74.4 vs. BC 87.4 min, $\mathrm{P}=0.006$ ), we found that $\mathrm{DN}$ cardioplegia still showed a significant reduction in the need for defibrillation compared with BC (DN $11.8 \%$ vs. BC $33.3 \%, \mathrm{P}=0.003$, Table 5). The average treatment effect estimation predicted a $30.4 \%$ reduced need for defibrillation with DN. PSM showed similar post-CPB LVEF between the two groups (DN $55.4 \%$ vs. $52.6 \% \mathrm{BC}, \mathrm{P}=0.880$ ).

\section{Discussion}

In this study, we showed that the use of anterograde singledose DN in isolated CABG surgery reduced CPB and aortic cross-clamp times, decreased the need for post-CPB 


\begin{tabular}{|lccc|}
\hline \multicolumn{2}{|c|}{$\begin{array}{l}\text { Table 5. Propensity Matched for Age, Sex, BMI, Preoperative Creatinine, Diabetes, STS 30-Day Risk Score, } \\
\text { Pre-CPB Ejection Fraction, and Cross-Clamp Time }\end{array}$} & Matched & \\
\cline { 2 - 4 } & BC (n=18) & DN (n=51) & \\
Cardiac function & & & \\
Post-CPB LVEF (\%) & $52.6 \pm 10.4$ & $55.4 \pm 9.6$ & 0.880 \\
Surgical data & & & \\
Need for defibrillation & $6(33.3 \%)$ & $6(11.8 \%)$ & $0.003^{* *}$ \\
Medical outcomes & & & \\
Hospital stay (days) & $6.7 \pm 3.5$ & $6.8 \pm 4.7$ & 0.773 \\
ICU stay (days) & $2.9 \pm 2.5$ & $2.9 \pm 4.6$ & 0.922 \\
Creatinine (mg/dL) & $1.21 \pm 0.47$ & $1.16 \pm 0.86$ & 0.303 \\
Atrial fibrillation & $3(16.7 \%)$ & $12(23.5 \%)$ & 0.787 \\
Readmission & $3(16.7 \%)$ & $5(9.8 \%)$ & 0.067 \\
All-cause death & $0(0 \%)$ & $0(0 \%)$ & - \\
MI & $0(0 \%)$ & $0(0 \%)$ & - \\
Stroke & $1(5.6 \%)$ & $2(3.9 \%)$ & 1.000 \\
\hline
\end{tabular}

Abbreviations as in Tables 1-3.

defibrillation, and provided similar short-term outcomes and comparable myocardial protection compared with a conventional multi-dose BC strategy. PSM enabled a more accurate assessment of post-CPB cardiac function and short-term outcomes, both of which may have been affected by the difference in cross-clamp time observed with DN compared with BC. PSM confirmed that the benefits in post-CPB rhythm stability and cardiac function associated with use of DN were most likely attributable to the properties of DN cardioplegia itself, and not caused by a reduction in cross-clamp time associated with the use of DN.

The mechanisms by which DN protects against myocardial ischemia, reperfusion injury, and calcium-induced hypercontraction are derived from specific components added to the cardioplegia solution, such as mannitol, magnesium, and lidocaine. Mannitol prevents hyperosmotic myocardial edema and free-radical damage, while magnesium acts to competitively block the influx of calcium ions into cardiomyocytes. ${ }^{1}$ Lidocaine acts as a modified depolarizing agent by inhibiting sodium channels to prevent calcium accumulation and counteract the effects of hyperkalemia. ${ }^{1}$ Indeed, O'Blenes and collaborators showed that, in rat heart isolates, $\mathrm{DN}$ was more effective than $\mathrm{BC}$ at suppressing diastolic calcium levels to prevent hypercontraction injury during reperfusion. ${ }^{3}$ Specifically, the lidocaine in DN reduces intracellular calcium by lessening the sodium 'window' current to modulate the sodium-calcium exchanger, ${ }^{3}$ thus allowing for superior functional recovery with higher peak cardiac output, systolic pressure, and stroke volume with DN. ${ }^{4}$

Although several recent studies have investigated the safety and effectiveness of DN in adult valvular surgery, 58 there is limited evidence to validate the use of $\mathrm{DN}$ in adult CABG surgery. Our data were concordant with those of a few other published studies showing that, for isolated CABG surgery, DN provided early clinical outcomes and myocardial protection comparable to that associated with the use of other conventional cardioplegia strategies. ${ }^{9-12}$ Yerebakan et al found a similar incidence of postoperative low cardiac output syndrome and in-hospital death after CABG using DN vs. multi-dose cold whole BC for patients with acute MI.11 Timek et al studied patients who underwent isolated $\mathrm{CABG}$ with either $\mathrm{DN}$ or $\mathrm{BC}$, and found that both strategies yielded similar postoperative troponin levels and inotrope requirements. ${ }^{10}$ It is not routine practice at our institution to measure molecular biomarkers of myocardial injury after cardiac surgery, because of the non-specific interpretation. Therefore, we specifically selected post-CPB LVEF and RV function measured by TEE as our metrics for assessing myocardial protection, as the immediate post-CPB period is when poor myocardial protection would be most apparent. We found in our propensity-matched cohort that DN and $\mathrm{BC}$ yielded similar post-bypass LVEF after CABG. Only one instance of RV dysfunction was observed immediately after weaning from $\mathrm{CPB}$, and this occurred in a patient who received $\mathrm{BC}$.

Another attractive quality of DN is its ease and efficiency of use, allowing operative teams to streamline the workflow of procedures requiring cardioplegic arrest. We used an anterograde-only technique, which reduces the risk of complications associated with retrograde cardioplegia catheter insertion (e.g., coronary sinus injury, bleeding from an additional surgical site). Although hearts with multivessel disease are traditionally arrested on CPB using combined anterograde and retrograde cardioplegia, both our results and those reported by Salinas et $\mathrm{al}^{\mathbf{9}}$ show that anterograde DN alone provides sufficient myocardial arrest and protection even in hearts with multivessel disease. In addition, our study, as well as those of Mishra et al ${ }^{12}$ and Yerebakan et al, ${ }^{11}$ found that the use of DN is associated with shorter CPB and aortic cross-clamp times compared with other cardioplegia strategies. The benefit of shorter operating times may not only translate to clinical benefits for the patient, ${ }^{13}$ but also improve the efficiency of operating room usage and decrease hospital costs.,, 10

Finally, we noted a significant reduction in the need for defibrillation using $\mathrm{DN}$ compared with $\mathrm{BC}$ in our unmatched groups, with $33.3 \%$ of the $\mathrm{BC}$ cohort requiring defibrillation compared with only $13.0 \%$ of DN cohort. After controlling for cross-clamp time, as well as other preoperative risk factors, the need for defibrillation was still significantly reduced from $33.3 \%$ with $\mathrm{BC}$ to $11.8 \%$ with DN. Our findings support similar results previously 
reported by Salinas et al, in which $42 \%$ of patients receiving $\mathrm{BC}$ required defibrillation after weaning off $\mathrm{CPB}$ compared with just $8 \%$ of patients who received DN. ${ }^{9}$ The avoidance of defibrillation not only improves the workflow of surgery, but also may provide benefit to the recovering post-surgical myocardium, as the association between open chest defibrillation during cardiac surgery and subepicardial necrosis is well-described. ${ }^{14}$ A novel aspect of our study involved the use of PSM to suggest that the association between DN and the reduced need for defibrillation was not due to a reduction in cross-clamp time but rather due to the properties of DN cardioplegia.

Of note, a randomized trial including adults undergoing first-time CABG using $\mathrm{DN}$ vs. whole $\mathrm{BC}$ is currently in progress, ${ }^{\mathbf{1 5}}$ and the preliminary results for 89 total patients suggest that a perfusion strategy using DN is safe and leads to a shorter aortic cross-clamp time, higher rate of return to spontaneous rhythm, and decreased requirement of intraoperative inotropic support. ${ }^{16,17}$ Whether DN will ultimately prove to be superior to other cardioplegia strategies for CABG surgery in terms of long-term clinical outcomes, myocardial protection, safety, efficiency, and cost, and therefore be justified to use as the default cardioplegia strategy for $\mathrm{CABG}$, remains a question of great interest.

\section{Study Limitations}

There are several limitations that must be noted. First, the relatively small number of patients may prevent us from resolving a statistically significant difference for our primary and secondary outcomes of interest. In addition, the retrospective nature and single-surgeon design of our study may introduce patient selection biases, although this was tempered by the fact that the surgeon was not given an option to choose the cardioplegia strategy used for each patient. The short-term follow-up, including for cardiac function, is a limitation in our study, as well as in all presently available studies, and should be addressed in the future.

\section{Conclusions}

Single-dose anterograde DN cardioplegia provided excellent myocardial protection with reduced need for intraoperative defibrillation, shorter bypass and cross-clamp times, and comparable early clinical outcomes for adult patients undergoing isolated CABG surgery compared with BC. The reduction in defibrillation requirements and shorter $\mathrm{CPB}$ and aortic cross-clamp times associated with DN may improve the efficiency and workflow of the procedure for the surgical team.

\section{Acknowledgments}

We thank Senior Biostatistician Hao He, PhD, for statistical review.

\section{Conflicts of Interest / Funding / Grants}

\section{IRB}

38337 (Approved 9/30/2016).

\section{References}

1. Matte GS, Nido PJ. History and use of del Nido cardioplegia solution at Boston Children's Hospital. J Extracorpor Technol 2012; 44: $98-103$.

2. Ad N. del Nido cardioplegia: Ready for prime time in adult cardiac surgery? J Thorac Cardiovasc Surg 2015; 149: 637-638.

3. O'Blenes SB, Friesen $\mathrm{CH}$, Ali A, Howlett S. Protecting the aged heart during cardiac surgery: The potential benefits of del Nido cardioplegia. $J$ Thorac Cardiovasc Surg 2011; 141: 762-770.

4. Govindapillai A, Hua R, Rose R, Friesen CH, O'Blenes SB. Protecting the aged heart during cardiac surgery: Use of del Nido cardioplegia provides superior functional recovery in isolated hearts. J Thorac Cardiovasc Surg 2013; 146: 940-947.

5. Takayama H, Soni L, Kalesan B, Truby LK, Ota T, Cedola S, et al. Bridge-to-decision therapy with a continuous-flow external ventricular assist device in refractory cardiogenic shock of various causes. Circ Heart Fail 2014; 7: 799-806.

6. Ramanathan R, Parrish DW, Armour TK, Brinster DR. Use of del Nido cardioplegia in adult cardiac surgery. Thorac Cardiovasc Surg 2015; 63: 624-627.

7. Ziazadeh D, Mater R, Himelhoch B, Borgman A, Parker JL, Willekes CL, et al. Single-dose del Nido cardioplegia in minimally invasive aortic valve surgery. Semin Thorac Cardiovasc Surg 2017; 29: 471-476.

8. Mick SL, Robich MP, Houghtaling PL, Gillinov AM, Soltesz EG, Johnston DR, et al. del Nido versus Buckberg cardioplegia in adult isolated valve surgery. J Thorac Cardiovasc Surg 2015; 149: 626-636.

9. Guajardo Salinas GE, Nutt R, Rodriguez-Araujo G. del Nido cardioplegia in low risk adults undergoing first time coronary artery bypass surgery. Perfusion $2017 ; 32$ : $68-73$.

10. Timek T, Willekes C, Hulme O, Himelhoch B, Nadeau D, Borgman A, et al. Propensity matched analysis of del Nido cardioplegia in adult coronary artery bypass grafting: Initial experience with 100 consecutive patients. Ann Thorac Surg 2016; 101: $2237-2241$.

11. Yerebakan H, Sorabella RA, Najjar M, Castillero E, Mongero $\mathrm{L}$, Beck J, et al. del Nido cardioplegia can be safely administered in high-risk coronary artery bypass grafting surgery after acute myocardial infarction: A propensity matched comparison. $J$ Cardiothorac Surg 2014; 9: 141.

12. Mishra P, Jadhav RB, Mohapatra CKR, Khandekar J, Raut C, Ammannaya GK, et al. Comparison of del Nido cardioplegia and St. Thomas Hospital solution: Two types of cardioplegia in adult cardiac surgery. Kardiochir Torakochir Pol 2016; 13: 295299.

13. Al-Sarraf N, Thalib L, Hughes A, Houlihan M, Tolan M, Young $\mathrm{V}$, et al. Cross-clamp time is an independent predictor of mortality and morbidity in low- and high-risk cardiac patients. Int J Surg 2011; 9: 104-109.

14. Kerber RE, Carter J, Klein S, Grayzel J, Kennedy J. Open chest defibrillation during cardiac surgery: Energy and current requirements. Am J Cardiol 1980; 46: 393-396.

15. Ad N. del Nido cardioplegia randomized trial. https://clinicaltrials. gov/ct2/show/NCT02442050 (accessed August 4, 2018).

16. Sinha P, Jonas RA. Time for a randomized prospective trial of single dose del Nido cardioplegia solution in adults. Perfusion 2016; 31: 34-37.

17. Ad N, Holmes SD, Massimiano PS, Rongione AJ, Fornaresio LM, Fitzgerald D. The use of del Nido cardioplegia in adult cardiac surgery: A prospective randomized trial. J Thorac Cardiovasc Surg 2018; 155: 1011-1018.

None. 